\title{
FICCIÓN, VERDAD, MENTIRA: BREVE HISTORIA DE UNA CANCIÓN DE NAVIDAD Y BOXEO EN TIERRA DEL FUEGO Y EL MUNDO (FINES DEL SIGLO XIX)
}

\author{
ALBERTO HARAMBOUR ${ }^{\circ}$
}

\section{RESUMEN}

Este artículo presenta una canción popular que sitúa su narración en la Tierra del Fuego de fines del siglo XIX, y analiza los componentes de veracidad, ficción y falsedad que presenta así como las condiciones de posibilidad de la historia cantada para aprehender las experiencias sociales del espacio colonial fueguino. A modo de hipótesis, se plantea que la circulación global de la música popular permite la acumulación y transformación de experiencias equívocas dentro de un mismo conjunto original de información.

PALABRAS CLAVE: literatura de viajes, Tierra del Fuego, música popular, folclore irlandés, microhistoria, historiografía.

\section{FICTION, TRUTH, LIES: SHORT STORY OF A CHRISTMAS AND BOXING SONG IN TIERRA DEL FUEGO (LATE $19^{\mathrm{TH}}$ CENTURY)}

\begin{abstract}
This article presents a popular song which situates its narrative in Tierra del Fuego during the second half of the nineteenth century. The vectors of conditions of possibility and historiographic veracity of the sung story for apprehending the social experiences of the colonial space are analyzed. As a hypothesis, we argue that the global circulation of popular music allows the accumulation and transformation of misleading experiences within the same original set of information.
\end{abstract}

KEY WORDS: travel literature, Tierra del Fuego, folk music, irish folklore, microhistory, historiography.

\section{CANCIONES DE LA EXPERIENCIA}

De la Tierra del Fuego colonial, cuyo tiempo se desplegó vertiginosamente con la fiebre del oro y la "invasión malvinera" de la década de 1880 (Braun 1985 , p. 79) y se cerró con la erradicación de la soberanía selknam y la consolidación de la economía ovina (Harambour, 2016, pp. 13-30), sabemos bastante: desde los detalles de las exploraciones 
hasta los regímenes de propiedad y sus resultados devastadores para la flora, fauna, suelo y pueblos colonizados. De la vida cotidiana de los hombres y mujeres para quienes ese tiempo fue el propio, un tiempo de descubrimiento, conquista, crimen y fundación bastante menos. Es posible reconstruir la trayectoria del avance de las ovejas, las disposiciones administrativas y algunos delitos, los informes oficiales y particulares sobre 'el problema del indio' y reconstruir las listas de bebidas y productos consumidos en las estancias $y$, algo menos, las entretenciones de quienes los consumieron. Hay algunos testimonios que refieren al consumo casi siempre excesivo de alcohol y a sus consecuencias, a la caza, para comer, o al simple asesinato masivo, por placer, de guanacos y aves, a la competencia y la apuesta en carreras de caballos, y a que sonó, en matrimonios, estancias y barcos la guitarra, el acordeón, el violín o la gaita (Childs, 1936; Finger, 1923, 1927, 1936a, 1936b; Harambour, 2016). De lo que en esos tiempos, en esos espacios de reunión se cantaba, sin embargo, no sabemos salvo generalidades.

El cantar público es una dimensión social de la vida en la que se conjugan las vivencias previas de unos sujetos con las de otros en un tiempo y un lugar lejano y compartido, distante de la experiencia previa de unos y otros. En los espacios de colonización tardía (ese poderoso ciclo de expansión imperial y nacional con el que se clausuraron los espacios independientes al capitalismo) quienes cantaban eran sujetos cuyas trayectorias han sido difíciles de imaginar para la historiografía: en la Tierra del Fuego se trató en su mayoría de hombres solos, salidos de pueblos de los Balcanes y Asturias, de las islas británicas (desde el Mar del Norte hasta Oceanía), provenientes del archipiélago de Chiloé, los conventos italianos y el

1 Entre estas obras destacan las crónicas de William Greenwood y las memorias y un diario de William Blain, aparecidas en ediciones realizadas por Campbell y GracePaz (2016) y Harambour (2016). A estos textos cabe sumar las más antiguas biografías de James Radburne (Childs 1936) y Edward Chace (Barrett \& Barrett, 1931).

2 La primera referencia la agradezco a Tomás Cornejo, quien compartió la cueca "El oro en Magallanes" publicada por Castillo (1900) en dos estrofas que se leen así: I / Vamos a Magallanes / Mi buen Teodoro, / Que allá por quintales / Se encuentra el oro. / Se encuentra el oro, hai, sí, / En lindas pepas / I a mas del que hai molido / Que no se cuenta, / Que no se cuenta, si /iQué lindo hallazgo! / Que habriendo [sic] un hoyito / Se llena un saco. / Yo hiria de carrera / Si cierto fuera. / II / Dicen que hai como infierno
Noroeste argentino, y de sus biografías individuales y colectivas sabemos poco. Ellos entonaban canciones de la experiencia, para tomar prestada la expresión de William Blake: de una experiencia colonial popular, transnacional y situada, o translocal (French, 2011), de la que sólo recientemente han emergido más referencias, aún escasas. Las experiencias tienden a permanecer en los territorios en los que se producen, transformándose creativamente de muchas formas, en la cultura local. En la "fueguina", sin embargo, con una alta tasa de reemigración (en el caso de los británicos, generalmente de regreso a la tierra natal), escasos vestigios permanecieron en la cultura popular. Sólo en los últimos años, a partir del hallazgo o redescubrimiento de fuentes perdidas $u$ olvidadas ha sido posible acceder a fragmentos de la vida cotidiana de las prácticas coloniales en Patagonia austral ${ }^{1}$. Las letras de canciones, en cambio, no existen historiográficamente cuando no quedan inscritas, que es el momento en que las cosas dichas pasan del campo de la oralidad a la escritura y de allí, como fuentes del saber, a la narración historiográfica (Ricoeur, 2004, pp. 191-192).

Hasta hace poco, tenía conocimiento de sólo dos canciones que hacían referencia al mundo del bajo pueblo colonial, mezclado en la aventura de la fiebre del oro y en los bares y prostíbulos de Punta Arenas. La primera apareció sólo parcialmente transcrita, al parecer, en un libro de 1993 de Mateo Martinic; la segunda, fue encontrada por Tomás Cornejo en una compilación editada en Santiago en el 1900. Ambas refieren a personas que, probablemente, pasaron a la Tierra del Fuego en algún momento, o contemplaron la posibilidad de enriquecerse alli, por lo menos. En ninguna de las dos, sin embargo, se hace referencia a la isla Grande ${ }^{2}$.

/Oro menudo, / Lo que si que el invierno / Es algo crudo. / Es algo crudo, hai, sí, / Eso no importa, / Que tener oro en saco / No es mala torta. / No es mala torta, hai, sí, / Que hombres sin chico / Sacando oro a destajo / Se han hecho ricos. / Vamos pronto Teodoro / A sacar oro". El otro caso fue transcrito por Mateo Martinic (1993, p. 39), al parecer de manera parcial, y en ella se cuenta de un recorrido por bares y prostíbulos de Punta Arenas. Respecto de la música de los grupos oligárquicos y religiosos existen más registros, sobre todo con la proliferación de la prensa a partir de 1894. Un reciente trabajo de Fonseca (2014) ha estudiado el funcionamiento de la orquesta de niños deportados en la Misión san Rafael en Dawson y las canciones que debían tocar. No se trata de temas locales, sin embargo, si no de un cancionero católico internacional. 
La primera canción cuya narración la situaba en la Tierra del Fuego anterior al 1900 apareció inscrita en el testimonio de un viajero inglés, que fue publicado en la década de 1920. Grande fue la emoción del descubrimiento particular, en medio de la más amplia que experimentaba con revisión de los cientos de páginas que él, Charles Finger, dejó inéditas o publicadas en Estados Unidos. Finger fue observador participante de la experiencia colonial popular, y su obra cubre diversos formatos escritos. Era un joven músico inglés, formado en la muy letrada tradición intelectual del socialismo Fabiano londinense, que a los 21 años arribó a la zona del Estrecho de Magallanes buscando convertirse en el primero en pararse donde no había pisado un hombre blanco (Finger, 1936a, p. 15). Aunque llegó tarde para serlo en el sur americano (era 1890), escribió sus diarios de vida regularmente, despachó correspondencia a medios de prensa y amistades, y continuó luego, desde otros territorios fronterizos en Norteamérica, escribiendo novelas, cuentos infantiles y de aventuras. En su inmensa obra, inédita en castellano, Patagonia y la Tierra del Fuego ocupan un lugar central: desde Arkansas o mirando hacia Australia, o bien narrando los viajes de Marco Polo, en Finger el extremo sur se convirtió en el polo magnético de sus referencias. Allá volvió siempre en la palabra escrita y nunca en persona: la experiencia social patagónicofueguina definió una matriz para la comprensión de la vida. Esto es, por cierto, muy significativo en sus memorias, y también en las dos recopilaciones musicales que publicó ${ }^{3}$.

En la obra de Finger destacan dos textos impresos con letras reunidas durante sus recorridos por el mundo. El primero en aparecer fue uno de los cientos de títulos publicados por los Blue Books, unos folletines de 10 centavos de dólar destinados a la entretención y educación popular editados por Emanuel Haldeman-Julius en Kansas. En Sailor Chanties and Cowboy Songs, o Cantos de marinos y canciones de cowboys (1923), Finger explica que su interés en el rescate de melodías y letras se definió en un momento crítico de su vida. Habiendo naufragado en la isla Villarino, en el extremo suroriental de la Tierra del Fuego, con otros seis

3 La tensión entre veracidad y ficción en los diarios de vida, memorias, cuentos y novelas de Charles Finger forma parte de un proyecto que me encuentro desarrollando en sobrevivientes se vieron obligados a cruzar la isla completa para llegar al Estrecho de Magallanes. Fueron días y noches de intemperie y hambre, comiendo sólo choritos (un marisco común en las costas del sur americano), pequeños frutos silvestres y huevos, y donde, "siempre mojados y miserables", de vez en cuando, alguno se animaba a entonar una vieja canción de marineros. Uno de ellos

De barba cana era, y muy, muy profano en toda ocasión, pero tenía un tenor plañidero y por una especie de hábito cantaba cada vez que descansábamos. Recuerdo una noche cuando estábamos agazapados en un ángulo en la base de un acantilado, como para protegernos de un viento penetrantemente helado, que cantó 'Amsterdam Maid,' una canción para nada educada, y con referencias un tanto libres, como la mayoría de las canciones de marineros son (Finger 1923, p. 5).

Las referencias libres no se detuvieron allí. El viejo "mintió descaradamente", según Finger, diciendo que su padre era el inventor de la melodía, y una vez que alcanzaron la civilización, al fin con un vaso de grog caliente (una mezcla de ron y agua azucarada) en las manos, le habría transmitido el deber que ahora Finger cumplía. Tenía que transcribir todos los temas que había escuchado, porque los tiempos estaban cambiando: desde la masificación de la navegación a vapor, cualquier empleado o raquero sirve como marinero, y sus viejas canciones van a desaparecer como los romanos. En la era del imperio todo estaba desvaneciéndose: ingleses recorrían el espacio fueguino antes desconocido, los selknam eran combatidos y deportados, y navegantes de los siete mares se encontraban en medio de la estepa. Entonces todavía, el viejo de barbas grises agregó, ningún hombre podía ser un buen marino a no ser que se supiera una canción para cada una de los trabajos a bordo. En adelante, estando sólo en la isla Isabel o entre gauchos en Argentina o cowboys en Texas, Finger iba a cumplir la tarea encomendada. Sailor Chanties fue la primera entrega de esas

la actualidad. Una buena introducción a esta discusión en Ginzburg (2010). 
canciones escuchadas en distintos lugares que, quizás por lo mismo, hacían referencia a la nostalgia por la patria chica que había quedado atrás o a las aventuras de otros hombres y mujeres: los esquiladores en Australia, Nueva Zelanda y Sud América, habiendo sido reclutados largamente por marineros desertores, usan canciones de marinos en el trabajo (27), anotaba Finger. De esas canciones, sin embargo, sólo una hacía referencia al tiempo-espacio de la colonización austral.

\section{UNA CANCIÓN EN LA LEJANA TIERRA DEL FUEGO}

Mientras el horizonte de posibilidades fronterizas se iba clausurando aceleradamente de norte a sur de América, en Estados Unidos la música y la literatura de cowboys, indios y bandidos aumentaba su alcance social y comercial (Fenster, 1989). Considerando el éxito de su folleto, sólo cuatro años después de publicada esa recopilación Finger lanzó un libro de tapas duras y 181 páginas, hermosamente ilustrado por Paul Honoré: Frontier Ballads volvió sobre algunas de las canciones transcritas antes, incluyó numerosas nuevas ("oídas y reunidas") y permitió al autor explayarse sobre los contextos en los cuales había escuchado estas "canciones de tierras sin ley". Por lo general se trata de ambientes socialmente diversos localizados en las fronteras de una civilización que recién terminaba su ciclo de más rápida y devastadora expansión sobre espacios y pueblos hasta entonces independientes (sin hombres blancos). Se trata de historias que, según Finger, eran cantadas a través de estos espacios de fractura y reacomodo cultural por cantineros y desertores, gauchos y marineros, polizontes y fugitivos, ovejeros y vaqueros, donde la fuerza del Estado era débil o inexistente. La Patagonia había sido, y aún más la Tierra del Fuego, uno de estos espacios sociales donde un hombre, antes de llegar, podía haber pasado por Montana, Sud África, Arizona, Australia y Canadá (Finger, 1927, p. 40); se trataba de sospechosos de piratería, de robo de ganado, de contrabando, de asaltos en los caminos, de desviaciones a la rectitud alli donde habia propiedad gubernamental. En palabras de Finger:

4 Las descripciones de William Blain sobre la celebración de las fiestas nacionales chilenas y de un matrimonio británico en Patagonia son muy ilustrativas de los ambientes en
En Patagonia todos los hombres tienen la misma altura', dice un proverbio moderno, y eso significa que ese es, o fue, un lugar donde no hay barreras de raza, nacionalidad, credo, o posición. Blancos, negros, pieles rojas; buscaplayas yanqui, escocés, inglés, falklander, chileno, argentino; desertores de barcos, graduados universitarios, ex lustrabotas, ovejeros, gauchos; caballero o diamante bruto, hombre pulido o colega -todos valen lo mismo en la cocina de una estancia (149).

En un matrimonio, en un 18 de Septiembre, en un barco o en un establecimiento de la industria ovina se encontraban muchos hombres y pocas mujeres de todas las procedencias y de todas las procedencias eran sus canciones ${ }^{4}$. De ellas, como señalara antes, sólo hay una que se desenvuelve por completo en la Tierra del Fuego. Se trata del poema narrativo de una pelea de box que tiene lugar en alguna parte de la isla Grande, en una noche de Navidad que podemos conjeturar se desarrolla a fines del siglo XIX -o más específicamente, a inicios de la década de 1890, cuando Finger estuvo allí y en tanto sujeto del imperio británico podía imaginar que para todos, como para el, el paisaje social colonial no presentaba 'barreras de raza'. Traducida al castellano puede leerse asi ${ }^{5}$ :

\section{Morrisey y el marinero ruso.}

1. A todos los hijos de Irlanda atención ahora les pido,

Mientras yo canto las alabanzas de un peleador fuerte y bravo.

Cuento el cuento como fue contado, por otros para mí,

De cómo el marino ruso peleó al bravo Jack Morrisey.

2. En Tierra del Fuego en Sud Ameri-kay, El ruso desafió a Morrisey y sobre él habló, 'Dicen que eres un luchador, y sostienes el cinturón yo veo,

que se desarrollaban experiencias musicales en el espacio colonial. Harambour (2016).

5 Ver Anexo I, la letra del original en inglés. 
Asi que ven, atrevido joven boxeador $y$ prueba un round conmigo.

3. Entonces habló el bravo Morrisey, ese corazón sólido y verdadero,

'Soy un galante irlandés a quien nadie puede sojuzgar,

Puedo derrotar a un yanqui, a un oso finés o ruso,

En honor del trébol, los laureles yo vestiré'.

4. Esas palabras enfurecieron al ruso en la lejana tierra extranjera,

Escuchar repetidas esas palabras el nunca, jamás soportaría,

'Oh Jack, eres liviano para mí, y no cometas ningún error.

Tendrás que entregarme el cinturón, o tu vida tomaré.

5. A pelear en la noche de Navidad estos héroes acordaron,

$Y$ miles vinieron de muy lejos, desde el norte del Chile lejano,

Los irlandeses y los rusos, sus corazones llenos de júbilo,

$Y$ muchos apostaron a que el oso ruso mataría al joven Morrisey.

6. Los marineros ahí, y los mineros, un banquero rico o dos,

$Y$ dagos $^{6}$ de las montañas, una partida muy feliz,

Con algunos de Valparaíso y del pueblo de Montevideo,

Para ver al luchador ruso arriar los colores irlandeses.

7. Los dos se apuntalaron, se pararon en el ring, una gloria que hay que ver,

Y Jack dibujó su cinturón atado alrededor con el verde trébol irlandés.

Con veinte mil dólares de apuesta para que todos los vieran,

El premio para el campeón que se quedara con la victoria.

6 "Dagoes" es una expresión despectiva utilizada para referirse a originarios de la península ibérica, extendida
8. Los dos se estrecharon las manos, miraron a su alrededor, y se entregaron a la pelea,

Que llenó a los viejos hijos de Erin de alegría al mirar esa gloriosa vista,

El ruso echó al suelo a Irlanda en el round once,

Y Morrisey yació sangrando sobre el suelo rocoso.

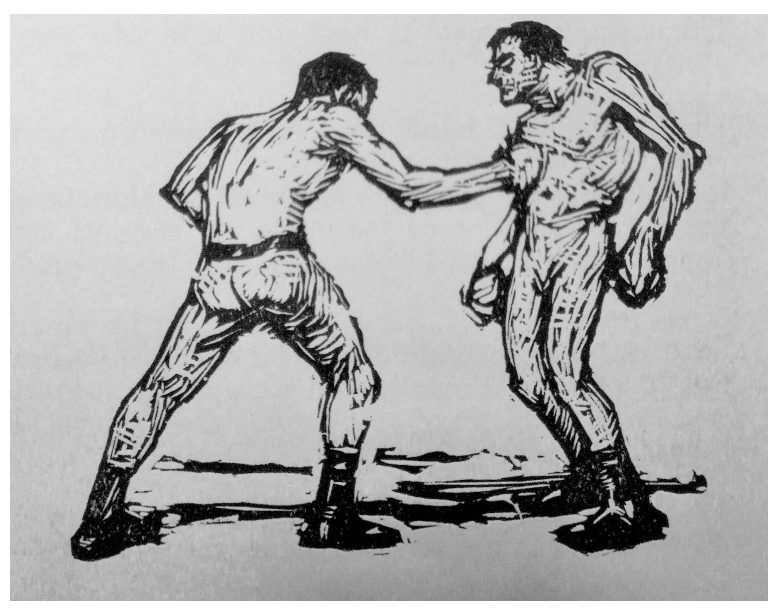

Fig. 1. Grabado de Paul Honoré (Finger, 1927, p. 45).

9. Un minuto y medio estuvo tirado antes de recobrar el aliento,

Con todos sus partidarios pensando que había caído muerto,

Pero Morrisey era resuelto y luchó para ponerse de pie,

$Y$ luchó bien duro y bravamente al ruso para hacerlo caer.

10. El irlandés comenzó apostando y ofreció cuatro a uno,

Tan pronto se hizo la oferta, otros hombres dijeron 'Hecho!'

Los luchadores hicieron sus asuntos y 32 rounds,

Una derecha en el hueso de la mandíbula mandó al suelo a Morrisey.

11. Luego fueron tres docenas de rounds, $y$ uno que fue caer y caer,

El ruso haciendo lo que pudo para noquear

también a italianos. Provendría del nombre común Diego, denominación portuguesa para marinero. 
a la vieja Irlanda,

Pero pronto llamó a su second y rogó por un trago de vino,

Entonces ese bravo héroe irlandés dijo, 'La batalla ahora es mía'.

12. El 38 fue el final, un round tan corto como agudo,

Morrisey con un tremendo golpe impactó a Rusia sobre el corazón,

El doctor con su lanceta, le abrió una vena,

Y dio como veredicto: 'él nunca va a volver a pelear'.

13. Ese fue Morrisey, el que le pegó a Thompson, al Yanqui Cliper también;

a Benicia Boy y a Shepherd, noblemente sometió.

Asi es que dejemos que todos llenen un vaso rebosante y beban un brindis o dos,

Por Jack el senador que boxea, quien hace las leyes por ti.

14. El peleó su camino a la fortuna, al dinero y a la fama,

Fue también un apostador, pero jugó limpio el juego,

El manejó a John C. Heenan y al fuerte viejo Bill Poole,

Y también venció a Sayers, el duro toro británico.

En la canción hay elementos que a quienes están familiarizados con la diversidad etno-nacional de los colonizadores de la Tierra del Fuego les resultarán conocidos. A mi sorpresa con el descubrimiento siguió la búsqueda de huellas de los nombres que se anotan: no pude encontrar en todo el archivo judicial ni administrativo del período ni a Hayes, ni a Heenan ni a Poole. Tampoco había rastros de ningún Morrissey, ni de ninguna de sus variables. En el comienzo de la narración, sin embargo, es el cantor quien dice que él cuenta el cuento como fue contado, por otros para mí. Esto nos sitúa más lejos de los hechos: alguien le contó esa historia al cantante, quien la convirtió en la canción escuchada por un escritor que permitió que llegara hasta nosotros en un libro. Conocemos por el escritor-testigo algo del cantor, pero no sabemos nada de quien primero la contó -el testigo directo. $\mathrm{Al}$ terminar la transcripción, el escritor agregaba una nota: Lo más entusiastamente que sea cantada, lo más grande que será su gozo. Cada persona que la escuche debe sentir que la canción le está dirigida a él personalmente. Aún sin tener acceso a este entusiasmo del volumen, por haberse reducido la canción entonada a una letra escrita, es posible percibir la épica de una pelea de box en una navidad en la Tierra del Fuego de la década de 1890, tiempo en el cual Finger la habitó y, al parecer, la sintió como dirigida a él. El combate, excepto por el exceso retórico de miles de asistentes y muchos miles de dólares en juego (lo más probable habría sido que allí y entonces la apuesta hubiese corrido en libras esterlinas), es perfectamente imaginable en un territorio de frontera en el que confluyeron cientos de hombres de las más diversas procedencias. Desde los puertos del Río de la Plata y del Chile central, irlandeses, finlandeses, británicos y yanquis, incluso algún potentado banquero (¿un estancierobanquero, un merchant-banker?), todos confluyen en el escenario colonial y hacen coincidir el relato poético con el saber factual o historiográfico nuestro y experiencial de Finger. Dicho de otra manera: lo que sabemos coincide con la experiencia que el escritor nos hace saber, quien está interesado en transmitirnos un testimonio fueguino que le parece coincidente a su vez con su propia experiencia. Estas coincidencias nos permiten afirmar la veracidad de la narración: salvo los que comprenderíamos como excesos retóricos, es perfectamente posible que sea verdad que esa pelea tuvo lugar en ese tiempo y en ese lugar.

La canción, sin embargo, había sido publicada 26 años antes que apareciera la versión de Finger, en un repositorio de antiguas canciones irlandesas y baladas, comprendiendo gemas patrióticas, descriptivas, históricas y humorísticas de la raza irlandesa. Esa compilación de Manus O'Conor apareció en Nueva York (1901) e incluyó decenas de temas que afirmaban la identidad irlandesa en un contexto de creciente poder en la Costa Este, luego de décadas de conflictivas relaciones interétnicas y subordinación a yanquis y a "toros u osos" sajones -quienes reemplazan en esta versión, titulada simplemente Morrisey y el ruso, a los fineses y "osos rusos". Este texto mantiene la estructura narrativa de 
los eventos, lo mismo que los nombres propios que aparecen hacia el final de la canción, lo mismo que otra versión que fue publicada por M. C. Dean en $1922^{7}$ y reeditada por Carl Sandburg en 1927 (el mismo año que la de Finger); también aparecen elementos diferentes en las versiones grabadas en 1947 por el gran recopilador Alan Lomax: en el oeste de Irlanda (Lydon 2012) y por el clásico del folclore irlandés Joe Heaney, a partir de 1960. Éstas comparten la mención inicial a la Tierra del Fuego, en Sud Amerikay; sin embargo, no contienen ninguna referencia a aquellos que llegaron a presenciar la pelea desde Valparaíso, Montevideo, o el norte del Chile distante; además, se indica que las provocadoras palabras iniciales de Morrisey enfurecieron al marinero ruso, aunque ya no en esa lejana tierra extranjera (frase que sólo figura en los textos de Finger y de Dean) si no que en yanquilandia, o la tierra yanqui ${ }^{8}$. Y otro detalle: el bravo Jack Morrisey de las versiones de Dean y de Finger es llamado John, o simplemente Johnny, el irlandés.

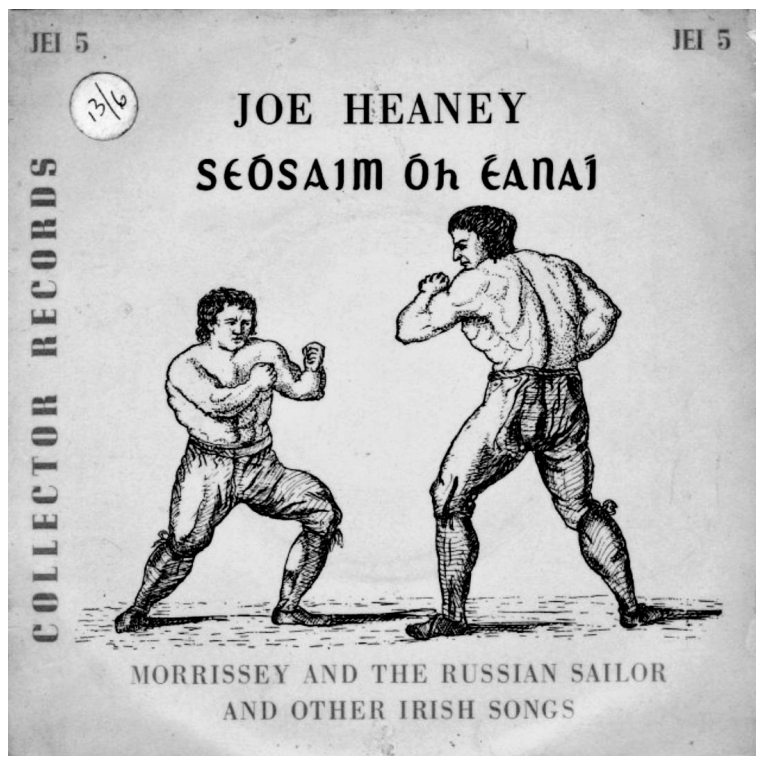

Fig. 2. Morrissey and the Russian Sailor fue grabada por primera vez en agosto de 1947 en Carna, costa atlántica irlandesa, por Johnny McDonagh para el segundo volumen de la recopilación de Alan Lomax titulada World Library of Folk \& Primitive Music. Imagen de la carátula del disco de Heaney, 1960.

7 La publicación de Dean, de escaso tiraje, apareció en Virginia conteniendo 150 canciones de hombres rurales, marineros, leñadores, soldados, hombres de los Grandes Lagos, ferroviarios, mineros.

\section{NOMBRES PROPIOS}

$\mathrm{Si}$ prescindimos de los puertos y las nacionalidades que aparecen al principio del poema y refuerzan el sentido heroico y brutal de un combate a muerte en las fronteras australes de la civilización, los nombres y sobrenombres de sus dos últimos párrafos recobran sentidos perdidos en los largos caminos sobre la mar que la canción recorrió, llevada en la voz de marineros anónimos. Morrisey, aunque llamado John, sigue siendo el que le pegó a Thompson y al Yanqui Cliper, a Benicia Boy y a Shepherd (Ovejero), a John Heenan y a Bill Poole, y también a Sayer. No se trata, sin embargo, de los apodos de desertores arrojados a las áridas playas de Patagonia, si no de los primeros campeones de box a puño limpio del Atlántico norte, todos sujetos de fama popular. John C. Heenan habría sido el primer deportista que se convirtió en una celebridad o superestrella de los Estados Unidos, según Duffy (2010); el tal Sayers corresponde al campeón de Inglaterra sobre el que existe amplia literatura (partiendo por Miles, 1866), con quien Heenan se enfrentó en Hampshire en 1860 para definir una suerte de primera corona del mundo, en un encuentro que ha sido considerado como la pelea que cambió al box para siempre -las reglas contemporáneas, al menos, se crearon luego de ella (Keating, 2010). Heenan era conocido como el Niño de Benicia o Benice Boy, por los muelles de California donde trabajó y peleó una vez desembarcado en ellos atacado por la Fiebre del Oro. William Poole, por su parte, es más conocido como Bill the Butcher, o El Carnicero, un célebre criminal de la Costa Este en el que se basó el personaje the Butcher Cutting, interpretado por Daniel Day-Lewis en la película Pandillas de Nueva York (Bordewich, 2002). El Cliper, a su vez, correspondería a James Yankee Sullivan, nacido como James Ambrose y también conocido como Frank Murray, temprano campeón estadounidense de boxeo. No hemos podido encontrar a ningún shepherd en esos tiempos y ambientes, pero sabemos que en Patagonia e Irlanda había muchos a quienes se

\footnotetext{
8 Varias versiones tradicionales, cantadas por Ewan MacColl y Joe Heaney desde la década de 1960, pueden escucharse en internet, lo mismo que versiones más recientes.
} 
llamaba ovejeros. Tampoco hemos identificado a ningún Jack Morrisey, ni como boxeador ni como senador estadounidense. El legendario Jack Morrissey, llamado así en las versiones de Dean (1922) y Finger (1927), y quien aparece descrito como quien hace las leyes por ti en todos los textos (salvo el de Dean), podría tratarse de John, sobre cuyas glorias pugilísticas existen otras baladas, entre las cuales la más difundida es "Morrissey and the Benicia Boy" (O'Conor 1901, p. 44).

Este John Morrissey fue un irlandés de Tipperary, que migró de niño a Nueva York, donde creció como asaltante y matón en las mafias que disputaban las apuestas, el contrabando y la prostitución y alli ganó fama y fortuna. Derrotando al Yanqui Sullivan, primero, y a John Heenan, en 1859, tuvo una breve y exitosa carrera pugilística que abandonó para dedicarse a la política. Con su retiro, Heenan viajó a Londres para enfrentar a Tom Sayers, en un combate que terminó en un controvertido empate con los asistentes y la policía sobre el cuadrilátero. Ello quedó relatado, además de en la prensa, en múltiples canciones populares (Miles, 1966, p. 1906). Pocos años antes, Morrissey había perdido una pelea contra El Carnicero Poole, y otros pocos años después y como parte de sus actividades habituales tomó parte en su asesinato, igualmente célebre. Las organizaciones criminales convirtieron a Morrissey en diputado dos veces y en senador, otras dos, representando a la maquinaria del partido Demócrata y del Tammany Hall, que incluía a la pandilla católica irlandesa de los Dead Rabbits (Nicholson, 2016). El senador que boxeaba, quien hace las leyes por ti falleció en mayo de 1878. Según Sandburg (1927, 398), nunca enfrentó a un marinero ruso, ni una Navidad ni un 10 de junio, fecha del combate según su versión; sobre el paso de Morrisey por la Tierra del Fuego no se pronuncia.

\section{EPÍLOGO}

Nada hay que nos indique que el combate entre John o Jack Morrissey y un marinero ruso sin nombre tuviera lugar, alguna vez, en la Tierra del Fuego. Dado que en distintos lugares del mundo se ha inscrito alguna variación de la canción, es del todo probable que Finger efectivamente la escuchara en alguna parte. Sobre las circunstancias en que la escuchó, sin embargo, hay un elemento que mueve a confusión. No existe (hasta donde sabemos) una isla Villarino en el extremo sur americano. Una que lleva tal denominación se encuentra en la desembocadura del Río Negro, 1.600 kilómetros al norte de la Isla de los Estados donde, a mediados de 1890, ocurrió el trágico naufragio de un buque de Liverpool procedente de Malvinas, el Sea Toller. Finger no aparece en la lista de sobrevivientes que publicó la prensa. En su diario de vida, que permanece sin ser publicado, anotaba por esas fechas que se encontraba buscando oro en la costa norte de la Tierra del Fuego (Finger, 1890). Si estos dos documentos son veraces, no sólo es falso que Finger compartiera las noches de hambre de los sobrevivientes: estaríamos en presencia de una mentira, en tanto es difícil creer que recordara como cierto un evento tan significativo como su propio naufragio. Sin embargo, sabemos que los náufragos denunciaron en Punta Arenas los malos tratos a los que fueron sometidos en el vapor Villarino, de la Armada argentina, cuando fueron rescatados ${ }^{9}$, y consiguieron algún apoyo, no sabemos por qué, del joven Finger en éste puerto. Los conoció, si sabemos, y estableció correspondencia con ellos (si hemos de creer en las anotaciones de su diario de vida). Bien pudo escuchar de alguno de ellos Morrisey and the sailor, aunque no fueran compañeros de naufragio.

En las "tierras sin ley" del mundo de fines del siglo XIX, de acuerdo con Charles Finger, las historias sobre peleas por premios eran casi tan populares como aquellas que narraban los derroteros de desertores, náufragos y buscavidas escapando del encierro de las leyes: las Batallas Decisivas de la Historia, en opinión de los casos duros, no fueron peleadas por Alejandro, Darío, o Grant si no por rudos boxeadores. Hombres sin mayor educación, escribía Finger, te pueden decir que Jim Figg fue el primer campeón mundial; que el primer premio peleado en Estados Unidos fue entre Jacob Hyer y Tom Beasley; que la pelea más larga fue entre Mike Madden y Bill Hays y duró 185 rounds y así. De allí, señalaba, la popularidad de esa maravillosa y emocionante letra del combate de Morrisey que

\footnotetext{
9 "Terrible story of shipwreck", The New Zealand Herald, 7 de febrero de 1891, suplemento, p. 2.
} 
encontramos en las costas de Irlanda, en el centro de Estados Unidos, en el oeste de Canadá y situada al sur de la Patagonia. Su relato es plausible para cualquier persona familiarizada con la irrupción ovina en la Isla Grande. Se trata de una experiencia si no verificable al menos creíble que, salvo por las referencias geográficas, bien podría estar situada en Australia o en Iowa, o en Tasmania, o en Namibia. El paisaje social que describe es tan "universal" como la experiencia colonial, situable en espacios donde las fronteras entre lo legal y lo criminal están mal definidas o poco importan protagonizada por británicos, donde confluyen sujetos de las más variadas procedencias y ocupaciones y las mujeres y los indígenas son borrados de la épica si no la padecen, pasivos. Estas fronteras de la civilización, desde Oceanía hasta América, también pueden situarse en el Five Points de Nueva York de la década de 1850, donde las delimitaciones culturales, étnicas y de clase estaban redefiniéndose en los muelles, el cuadrilátero y el congreso. No se puede descartar, por lo mismo, la ocurrencia de una pelea navideña entre un ruso y un irlandés en la Tierra del Fuego de fines del siglo XIX, cuando Charles Finger recorrió la isla en varias direcciones, varias veces. La historia entonada como canción era coherente con la experiencia dialéctica, aunque no lo sea científica o factualmente (Jay, 2003), de los sujetos que llegaron a escucharla en otras tierras o en esas (si es que así sucedió). Se trata, por tanto, de una canción tan fueguina como neoyorquina o irlandesa, en la medida en que por esos territorios pasó la trayectoria global de hombres altamente móviles: una canción que ha ido por todo el mundo, a Australia, Nueva Zelandia, Patagonia y donde sea que se cría ganado, señalaba Finger sobre otra canción, "The dying cowboy" (Finger, 1923, p. 57).

Nos encontramos frente a una "inscripción" variable, de una canción-fuente abierta para la reconfiguración y el reconocimiento identitario, que canta a la nostalgia de un espacio-tiempo fuera de la ley y con leyes propias, especialmente la del hombre más fuerte entre iguales libres. Finger es, en cuanto a ésta canción, un testigo privilegiado. Incluso si la escuchó décadas después de 1890, en Arkansas, y allá le recordó una pelea que, aunque no tuvo lugar en la Tierra del Fuego, perfectamente podría haber ocurrido en 1890, una noche de navidad o un 10 de junio. Si los hechos y personajes de la canción son ficticios, cualquier semejanza con la realidad no es pura coincidencia. Ella persiste en diferentes lugares del mundo en diferentes versiones, unidas por el hilo de una experiencia compartida por sujetos que no se conocieron y quizás nunca conocieron la Tierra del Fuego. Cantadas, impresas o grabadas, esas versiones, variaciones o anomalías se han constituido parcialmente superpuestas, en saberes y creencias arraigadas en un pasado lejano y no documentado. Estas anomalías conjuntivas, como las denomina Ginzburg (2017, pp. 116117), conforman un saber histórico efectivo: si la pelea ocurrió o no es menos importante que el hecho de la presunción de su ocurrencia (Williams, 2010, pp. 197-198), y nos permite aproximarnos a las experiencias sociales en un territorio de colonización donde ello era perfectamente narrable como verdad, como ficción o como mentira. Sea una cosa u otra, la canción es la primera localizada en Tierra del Fuego.

\section{AGRADECIMIENTOS}

El autor agradece el apoyo del Centro IDEAL (Fondap), del que es investigador asociado; el descubrimiento y envío de la cueca "El oro en Magallanes" por el historiador José Tomás Cornejo, del que es admirador; y el entusiasmo por este texto de Valentina Espinoza, de quien es su enamorado.

\section{BIBLIOGRAFÍA}

Bordewich, F (diciembre 2002). "Manhattan Mayhem". Smithsonian Magazine, diciembre. Disponible en http://www.smithsonianmag.com/arts-culture/ manhattan-mayhem-72604720 (acc. 10 de marzo de 2017).

Davenport, T. (2005). Little Blue Book Handlist. Corvallis: Haldeman-Julius Collectors Club.

Dean, M. C. (1922). Flying Cloud. And the hundred and fifty other Old Time Songs and Ballads of outdoor men, sailors, lumber jacks, soldiers, men of the Great Lakes, railroadmen, Miners, etc. Virginia: The Quickprint.

Duffy, P. (5 de mayo de 2010). America's first sports superstar. Slate. Disponible en http://www.slate.com/articles/ sports/sports_nut/2010/05/americas_first_sports_ 
superstar.html (acc. 12 de marzo de 2017).

El oro en Magallanes - Cueca, en V. Castillo, El Festivo. Primer cuaderno de poesías populares, Santiago, Centro Editorial La Prensa, 1900, pp. 9-11. Col. Domingo Edwards, Archivo Central Andrés Bello, Universidad de Chile.

Fenster, M. (1989). Preparing the audience, informing the performers. John Lomax and Cowboy songs and other frontier ballads. American Music 7 (3), 260-277. Disponible en https://www.jstor.org/ stable/3052074?seq=1\#page_scan_tab_contents (acc. 13 de marzo de 2017).

Finger, C. J. (1890). Diary, 1890. University of Arkansas Libraries-Special Collections, Charles Finger Papers, Series 2, Box 4.

Finger, C. J. (1923). Sailor Chanties and Cowboy Songs. Girard: E. Haldeman-Julius.

Finger, C. J. (1927). Frontier ballads. Songs from lawless lands. Heard and gathered by C. J. Finger. Woodcuts by P. Honore. London: William Heinemann.

Finger, C. J. (1936a). Valiant Vagabonds. Kansas: D. Appleton Century.

Finger, C. J. (1936b). Forty years in Patagonia. The Saturday Review, 1 de agosto de 1936, p. 7

Fonseca, N. (2014). Servid al señor con alegría'. Música en la Misión san Rafael Arcángel (1889-1911). Magallania, 42(2), 23-39.

French, J. (2011). Another world history is possible. Reflections on the translocal, transnational, and global. En L. Fink (Ed.), Workers Across the Americas: The Transnational Turn in Labor History (pp. 3-11). New York: Oxford University Press,

Ginzburg, C. (2010 [2006]). El hilo y las huellas. Lo verdadero, lo falso, lo ficticio. Buenos Aires: Fondo de Cultura Económica.

Ginzburg, C. (2017). Anomalías conjuntivas: una reflexión sobre los hombres lobo. Revista de Estudios Sociales, 60, 110-118.

Grace Paz, G., \& Campbel, D. (2016). Patagonia bravía. Naturaleza, vidas y aventuras. Memorias originales del baqueano William H. Greenwood. Ilustrado por Julieta Fernández. Punta Arenas: Imprenta Maval.

Harambour, A. (2016). Viaje a las colonias. Memorias de un ovejero escocés en Malvinas, Patagonia y Tierra del Fuego (1878-1898). Traducción de M. Azara y A. Harambour. Santiago: DIBAM-Centro de Investigaciones Diego Barros Arana.

Jay, M. (2003). La crisis de la experiencia en la era postsubjetiva. Traducción de E. Sabrowsky. Santiago: Ediciones Universidad Diego Portales.

Keating, F. (2010). Heenan v. Sayers. The fight that changed boxing forever, The Guardian, 24 de abril de 2010. Disponible en www.theguardian.com/sport/ blog/2010/apr/14/john-heenan-tom-sayers-boxing (acc. 6 de marzo de 2017).

Lydon, M. (2012). Lomax in Ireland: fieldwork, commercial recordings and 'great remembers'. Galway: National University of Ireland.

Sandburg, C. (1927). The American Songbag. New York: Harcourt, Brace and Company.

The Joe Heaney Archives (2015). Morrissey and the Russian Sailor, http://www.joeheaney.org/en/morrissey-andthe-russian-sailor (acc. 15 de marzo de 2016)

Martinic, M. (1993 [1986]). Nogueira el Pionero. Punta Arenas: Universidad de Magallanes. Disponible en http://www.memoriachilena.cl/602/w3-article-8885. html\# (acc. 7 de diciembre de 2015).

Miles, H. D. (1866). Tom Sayers, sometime champion of England, his life and pugilistica career. London: S.O. Beeton.

Miles, H. D. (1906). Pugilistica. The History of British boxing. Vol. III. Edinburgh: John Grant. Disponible en https:// archive.org/details/pugilisticahist02milegoog (acc. 12 de febrero de 2016).

Nicholson, J. (2016). The notorious John Morrissey: how a bare-knuckle brawler became a congressman and founded Saratoga race course. Kentucky: University Press of Kentucky.

Ricoeur, P. (2004). La memoria, la historia, el olvido. Trad. Agustín Neira. Buenos Aires: Fondo de Cultura Económica.

Williams, S. (2010). Focus: Irish Traditional Music. Routledge: Nueva York-Oxon, 2010.

\section{ANEXO I.}

Morrissey and the Russian Sailor

(Finger, 1927, pp. 44-48)

1. O al ye sons of Ireland, attention now I crave,

While I do sing the praises of a fighter bold and brave.

I tell the tale as it was told by others unto me,

Of how the Russian sailor fought the brave Jack Morrisey. 
2. In Tierra del Fuego in South Ameri-kay,

The Russian challenged Morrisey and unto him did say,

'They say you are a fighting man and hold the belt I see,

So come my bold young fighter and try a round with me.'

3. Then up spoke brave Morrisey, that heart so stout and true,

'I am a gallant Irishman who no one can subdue,

For I can whip a Yankee, a Finn or Russian bear,

In honour of the shamrock, I will the laurels wear.'

4. Them words enraged the Russian in that far foreign land,

To hear them words repeated he'd never, never stand,

'O Jack, you are to light for me, and don't make no mistake.

You'll have to hand to me the belt, or else your life I'll take.

5. To fight over a Christmas day these heroes did agree,

And thousands came from far away, from north of far Chile,

The Irish and the Russians, their hearts filled full of glee,

And many bet the Russian bear would kill young Morrisey.

6. The sailors there and miners, a banker rich or two,

And dagoes from the mountains, a very happy crew,

With some of Valparaiso and Montevideo town,

To see the Russian fighter take the Irish colours down.

7. They both stripped off, stepped in the ring, a glory to be seen,

And Jack he wrote his belt bound round with Irish shamrocks green.

For twenty thousand dollars the stake for all to see,

The champion's prize to have and hold who won the victory.

8. They both shook hands and looked around and then fell to the fight,

It filled old Erin's sons with joy to see the glorious sight,

The Russian he floored Ireland in the eleventh round,

And Morrisey he lay bleeding upon the rocky ground.

9. A minute and a half he lay before he got his breath,

With all his backers thinking he'd fallen to his death,

But Morrisey was manful and struggled to his feet,

And fought right hard and gamely the Russian for to beat.

10. The Irish started betting and offered four to one,

No sooner was the offer, when other men said 'Done!'

The fighters stuck to business and thirtysecond round,

A right on the jaw bone brought Morrisey to the ground.

11. Then for three dozen rounds and one twas fall and fall about,

The Russian doing what he could to knock old Ireland out,

But soon he called his second and begged a drink of wine,

When that brave Irish hero said, 'The battle now is mine'.

12. The thirty-eight it was the end, a round both short and sharp,

Morrisey with a fearful blow hit Russia o'er the heart,

The doctor with his lancet, he opened up a vein.

And gave it as a verdict, 'He'll never fight again'. 
13. 'Twas Morrisey who beat Thompson, the Yankee Clipper too;

Benicia Boy and Shepherd, he nobly subdue.

So let all fill a brimming glass and drink a toast or two,

To Jack the boxing senator who makes the laws for you.

14. He fought his way to fortune, to money and to fame,

He also was a gambler, but square he played the game,

He handled John C. Heenan and burly old Bill Poole,

And also conquered Sayers, the hardy British bull.

Note: The more deliberately this is sung, the greater will be the enjoyment.

Each hearer must feel that the song is addressed to him personally." 